\title{
Green cardamom plus low-calorie diet can decrease the expression of inflammatory genes among obese women with polycystic ovary syndrome: a double-blind randomized clinical trial
}

\author{
Sahar Cheshmeh ${ }^{1}$ - Maysa Ghayyem ${ }^{2} \cdot$ Firoozeh Khamooshi $^{1} \cdot$ Neda Heidarzadeh-Esfahani ${ }^{1} \cdot$ Negin Rahmani $^{3}$. \\ Niloofar Hojati ${ }^{1}$. Elaheh Mosaieby ${ }^{4}$. Shima Moradi ${ }^{5}$ iD $\cdot$ Yahya Pasdar $^{2}$
}

Received: 15 February 2021 / Accepted: 20 May 2021 / Published online: 31 May 2021

(c) The Author(s), under exclusive licence to Springer Nature Switzerland AG 2021

\begin{abstract}
Purpose Polycystic ovary syndrome (PCOS) is an endocrine disorder and a common cause of infertility among women that is associated with low-grade inflammation. Therefore, the current randomized, double-blind, placebo-controlled clinical trial was conducted to assess the effects of green cardamom supplementation on the serum level of inflammatory markers and their gene expression among obese women with PCOS.

Methods We included 194 obese women with PCOS and administered low-calorie diet to all of them. These subjects were randomly divided into two groups including the intervention group with $3 \mathrm{~g} /$ day green cardamom $(n=99)$ and the placebo group $(n=95)$. Anthropometric indices, androgen hormones, and inflammatory factors [tumor necrosis factor- $\alpha(\mathrm{TNF}-\alpha)$, interleukin 6 (IL-6), and C-reactive protein (CRP)] were assessed before and after the 4-month intervention. TNF- $\alpha, I L-6$, and $C R P$ gene expression levels were measured using reverse transcription-polymerase chain reaction (RT-PCR) method.

Results Anthropometric indices were improved in both studied groups $(P<0.001)$. Among androgen hormones, luteinizing hormone, androstenedione, and dehydroepiandrosterone were significantly decreased $(P<0.001)$, and follicle-stimulating hormone was significantly increased $(P<0.001)$ in the green cardamom group. Our findings showed that TNF- $\alpha$, IL-6, and CRP serum levels were significantly decreased after the intervention with green cardamom plus low-calorie diet $(P<0.001)$. In addition, the expression levels of $T N F-\alpha$ and $C R P$ genes were significantly decreased in the intervention group $(P<0.001)$. Conclusions The present study supports the beneficial anti-inflammatory effect of green cardamom on the inflammatory state in PCOS women.

Level of evidence Level I: randomized clinical trial.

Trial registration This trial was registered with the Iranian Clinical Trials Registry (registration number: IRCT20200608047697N1). 1 August, 2020; https://www.irct.ir/trial/48748.
\end{abstract}

Keywords Polycystic ovary syndrome $\cdot$ Tumor necrosis factor- $\alpha \cdot$ Interleukin $6 \cdot$ C-reactive protein $\cdot$ Gene expression . RT-PCR

Shima Moradi

Shima.moradi@kums.ac.ir

1 Student Research Committee, School of Nutritional Sciences and Food Technology, Kermanshah University of Medical Sciences, Kermanshah, Iran

2 Resident of Internal Medicine, School of Medicine, Tehran Azad University of Medical Sciences, Tehran, Iran

3 Julius Maximillian University of Wuerzburg, Wuerzburg, Germany
4 Department of Molecular Biology Sciences, Mazandaran University, Mazandaran, Iran

5 Department of Nutritional Sciences, Research Center for Environmental Determinants of Health (RCEDH), Health Institute, Kermanshah University of Medical Sciences, Kermanshah, Iran 


\section{Introduction}

Polycystic ovary syndrome (PCOS) is a common endocrine disorder and is one of the most common causes of infertility in women [1]. The disorder affects $6-10 \%$ of women during reproductive age and is characterized by ovarian dysfunction and androgen hormone imbalance. The symptoms include hirsutism, acne, menstrual irregularities, infertility, obesity, and insulin resistance [2]. Some studies have shown that chronic low-grade inflammation is seen in women with PCOS in which its presence worsens this condition $[2,3]$.

PCOS etiology is not yet known, but systemic inflammation appears to be involved in the pathogenesis of the disease [4]. Obesity exacerbates the symptoms of hyperandrogenism and creates a vicious cycle that promotes PCOS [5]. Abdominal obesity in particular increases the production of inflammatory cytokines by increasing the adipose tissue in the central regions of the body [6]. Serum levels of inflammatory factors including tumor necrosis factor- $\alpha$ (TNF- $\alpha$ ), Interleukin 6 (IL-6), and C-reactive protein (CRP) are increased in these patients compared to healthy women of the same age and weight [7]. Evidence confirms that the overexpression of TNF- $\alpha$ gene is associated with the proliferation of adipose tissue that leads to insulin resistance and is also involved in follicular atresia [7, 8]. $I L-6$ gene is located in human chromosome 1q21 and is responsible for IL-6 production [9]. IL-6 is also an important pro-inflammatory factor that promotes cardiovascular disease, diabetes, and allergic reactions and affects the process of ovulation, implantation and fertilization [10]. CRP is encoded by $C R P$ gene and is an acute-phase protein that is synthesized in hepatocytes by IL-6 stimulation [11]. Evidence suggests that increased TNF- $\alpha$, IL- 6 , and CRP levels in PCOS patients indicate a low-grade chronic inflammation $[1,12]$. Therefore, dietary interventions aimed at weight loss and consequent reduced inflammation seem to be an appropriate strategy in these patients.

Green cardamom is a capsule of a dried fruit that belongs to the Zingiberaceae family and is also called Elettaria cardamomum (L.) Maton [13]. This traditional aromatic plant has antibacterial and anti-inflammatory roles and both its extract and fruit are attractive to pharmacists [14]. Green cardamom is rich in polyphenols and flavonoids such as lutein, anthocyanin and quercetin, which give the plant its antioxidant and anti-inflammatory properties [15]. Since PCOS patients suffer from low-grade systemic inflammation and this inflammation worsens the progression of their disease, and due to the anti-inflammatory role of green cardamom, the present study was conducted to evaluate the effects of green cardamom on the serum levels of inflammatory factors and their gene expression among obese women with PCOS who are on low-calorie diet.

\section{Method}

\section{Study design}

This study was designed according to CONSORT statement [16] as a randomized, double-blind, placebo-controlled clinical trial in which the effect of green cardamom supplementation on inflammatory markers and gene expression was assessed among obese women with PCOS. Sample size was calculated to be 75 subjects for each group based on a previous study [17] (95\% power and 5\% significance). However, we allocated 100 subjects to each group to increase the accuracy. The study was ethically approved by the Ethics Committee of Kermanshah University of Medical Sciences (Ethical NO: IR.KUMS.REC.1399.375) and registered with the Iranian Clinical Trials Registry (registration number: IRCT20200608047697N1). Written consent form was obtained from all studied subjects.

\section{Participants, recruitment, and randomization}

In the current study, PCOS patients were selected from gynecology and female infertility clinics in Kermanshah, western Iran. Eligible individuals for our study were PCOS patients who were diagnosed according to Rotterdam criteria if there are at least two factors: (1) oligomenorrhea or amenorrhea; (2) biochemical or clinical signs of increased androgens in the blood; and (3) having polycystic ovaries based on ultrasonography report. Other inclusion criteria were the age range (18-45 years), obesity [based on body mass index $(\mathrm{BMI}) \geq 30 \mathrm{~kg} / \mathrm{m}^{2}$ ], not being pregnant and breast-feeding, and willingness to participate in this study. Women with cardiovascular diseases, thyroid disorders, autoimmune diseases, gastrointestinal, mental disorders, asthma, and chronic bronchitis were excluded from the study. Moreover, women who consumed any dietary supplements, and those with allergies to green cardamom, green cardamom tea, and green cardamom products were not included in the study. At the beginning, we assessed 219 subjects for eligibility. Twenty subjects were excluded due to infection with coronavirus, being non-native, and pregnancy. Eventually, using the random number table method, 199 subjects were randomly allocated to two groups of cardamom $(n=100)$ and placebo $(n=99)$ (Fig. 1).

\section{Intervention}

We administered a low-calorie diet to all participants due to ethical considerations such as weight loss is part of the treatment protocol for these patients. For this purpose, patients' diet was adjusted to reduce 400-500 kcal per day of the daily calorie intake based on the adjusted ideal 


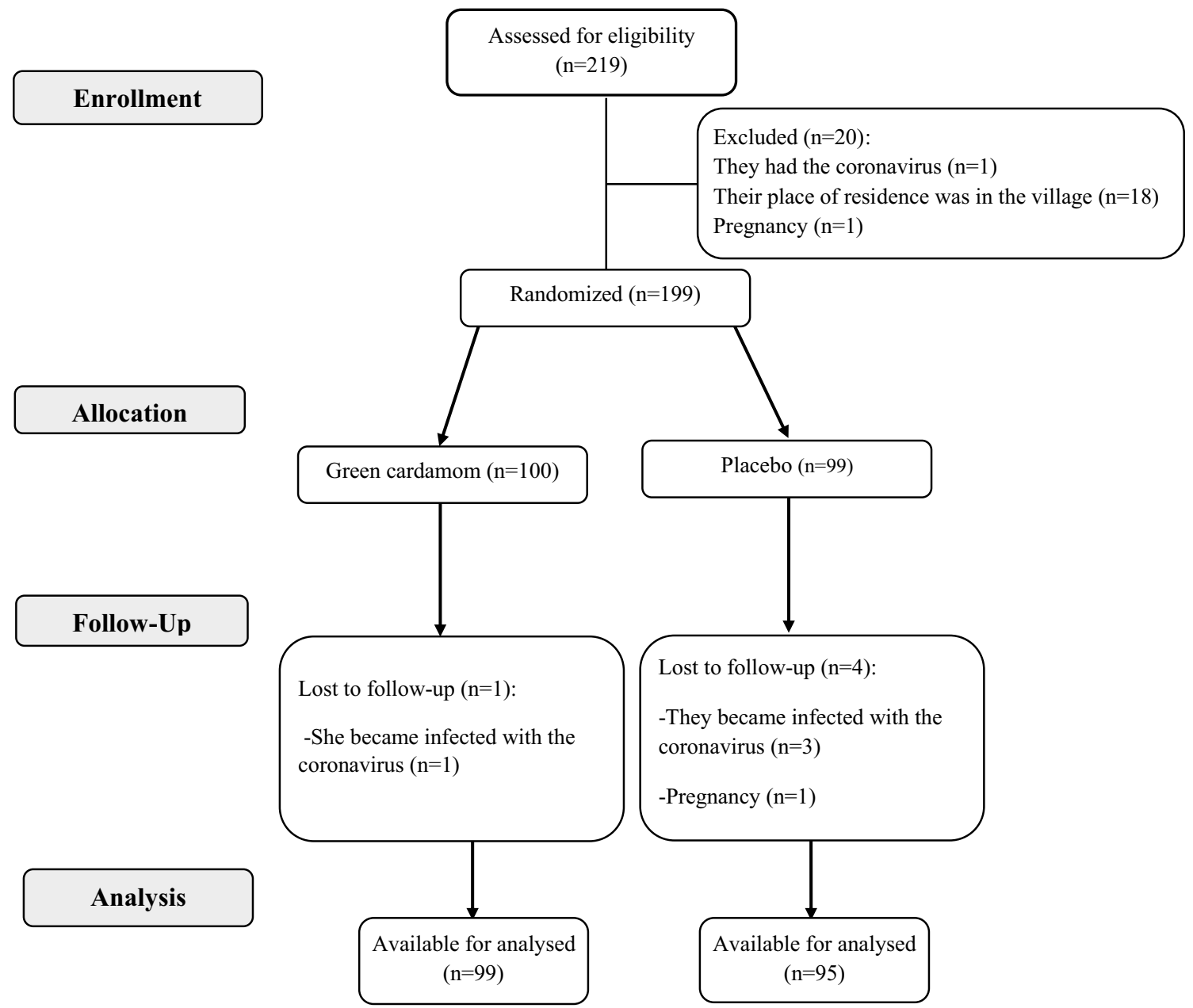

Fig. 1 CONSORT flowchart of study

weight. Additionally, $3 \mathrm{~g}$ cadmium powder was administered with each meal to the intervention group (three $1000 \mathrm{mg}$ green cardamom capsules, Karen Co, Iran) for a duration of 16 weeks. Daily consumption of green cardamom with this dose did not lead to poisoning and improved inflammatory status, lipid and glycemic profiles in previous studies [17-19]. In addition to the low-calorie diet, the placebo group received three capsules containing starch powder (with the same shape, color and size of cardamom supplement) daily throughout the intervention period. None of the researchers and subjects knew the content of the capsules until the end of the study and these capsules were coded by the company. Due to the COVID19 crisis, we used social media and held online training sessions. In order to encourage the participants to adhere to the diet and supplementation, we drew lots among them and gave them a prize every month.

\section{Measurements}

Demographic information, physical activity, and dietary intake of all subjects were collected by a trained dietitian. The international physical activity questionnaire (IPAQ) short form was used to assess physical activity. All subjects completed a 3-day food record ( 2 weekdays and 1 day in the weekend) to evaluate their energy and macronutrients intake before and after the intervention. NUTRITIONIST IV software was applied to measure the energy and nutrients intake based on the United States Department of Agriculture Food Composition Table, which was modified for Iranian foods [20]. The subjects were asked not to change their physical activity during the period after the administration of low-calorie diet. Other data related to the anthropometric indices, biochemical indices, and 
the expression of inflammatory genes before and after the intervention were assessed as follows.

\section{Anthropometric indices}

Bioelectrical impedance analysis (Inbody 770, Inbody Co, Seoul, Korea) was used to measure the weight and body fat mass (BFM) in standing position with light clothing. A wall-mounted stadiometer (Seca, Hamburg, Germany) was used to measure the height in the standing position without shoes. The weight $(\mathrm{kg})$ divided to height square $(\mathrm{m})$ was used as BMI $\left(\mathrm{kg} / \mathrm{m}^{2}\right)$. Waist circumference (WC) was measured by a non-stretched and flexible tape at the level of the iliac crest [21].

\section{Biochemical indices}

Ten milliliter of fasting venous blood was collected from all women at the beginning of the follicular phase (3rd day of the menstrual period). The samples were centrifuged, and serum was stored at $-80{ }^{\circ} \mathrm{C}$ until analysis. Luteinizing hormone ( $\mathrm{LH})$ and follicle-stimulating hormone (FSH) were measured by radioimmunoassay (RIA) technique. Other androgen hormones including thyroid stimulating hormone (TSH), testosterone, prolactin, androstenedione, and dehydroepiandrosterone (DHEA) were measured by Monobind kit and SGHB by ELISA.

\section{Expression of inflammatory genes}

We kept the blood samples in coated vials of ethylenediaminetetraacetic acid (EDTA) to assess the expression of $T N F-\alpha, I L-6$ receptor $(I L-6 r)$, and $C R P$ genes. Peripheral blood mononuclear cells (PBMC) were separated during density gradient centrifugation (Ficoll-paque, Miltenyi Biotec GmbH, and Germany) by Ficoll-Histopaque solution gradient (Ficoll-paque, Miltenyi Biotec $\mathrm{GmbH}$, and Germany). Total RNA from PBMC cells was extracted by Trizol Reagent kit (YTzol pure RNA, Iran) and $1 \mu \mathrm{g}$ of the extracted RNA was used for complementary DNA (cDNA) synthesis by Prime Script-RT Reagent kits (Takara Bio Ink. Tokyo, Japan). We purchased special primers from Metabion (Metabion, Germany) whose sequences are shown in Table 1. Data were normalized to the rate of $18 \mathrm{~S}$ rRNA expressing as housekeeping gene and then calculated based on fold change formula. All samples were conducted in three versions.

\section{Statistical analysis}

SPSS software (SPSS Inc. Chicago, IL, USA version 19) was used to perform the statistical analysis. The normality of the data was determined by Kolmogorov-Smirnov test. We used
Table 1 Primers sequences for RT-PCR amplification

\begin{tabular}{ll}
\hline $\begin{array}{l}\text { Gene name and } \\
\text { symbol }\end{array}$ & Sequence $\left(5^{\prime} \rightarrow 3^{\prime}\right)$ \\
\hline TNF- $\alpha$ & F: 5'-GTCAACCTCCTCTCTGCCAT-3' \\
& R: 5'CCAAAGTAGACCTGCCCAGA-3' \\
IL-6 & F: 5'-GGTACATCCTCGACGGCATCT-3' \\
& R: 5'-GT GCCTCTTTGCTGCTTTCAC-3' \\
CRP & F: 5'-TTTTCTCGTATGCCACCAAG-3' \\
& R: 5'-TTTCC AATGTCTCCCACCAG-3' \\
18 s rRNA & F: 5'-ACCCGTTGAACCCCATTCGTG A-3' \\
& R: 5'-GCCTCACTAAACCATCCAATCGG-3'
\end{tabular}

$F$ forward, $R$ reverse

descriptive statistics to report the characteristics of the subjects. Chi-square test was used to determine the difference between the qualitative variables of the two groups. Mann-Whitney $U$ test and independent sample $T$ test were used to evaluate the difference in dietary intake, gene expression, and anthropometric and biochemical indices between the two groups. The difference between the mentioned variables within the studied groups was evaluated using paired samples $T$ test or Wilcoxon test. The expression of inflammatory genes was described in excel software (Microsoft software, 2010). $P$ value less than 0.05 was considered significant.

\section{Results}

We excluded five subjects from our study due to infection with the coronavirus $(n=4)$ and pregnancy $(n=1)$. Overall, 194 subjects participated in this study until the end (Fig. 1).

We evaluated the basic characteristics of all subjects including age, physical activity, marital status, weight, BMI, $\mathrm{WC}$, and BFM and we found no difference in these variables between the two studied groups before intervention. Furthermore, we assessed their calorie and nutrient intake and we found no difference between the two studied groups before and after intervention. Since we administered the low-calorie diet to all the subjects, there was a significant difference between the amount of calorie and nutrient intake within each of the studied groups before and after intervention (Table 2).

All the women included in this study had ovarian cysts diagnosed by ultrasonography. After the administration of green cardamom and low-calorie diet, $40.6 \%$ of PCOS patients had no cyst in ultrasonography, while in the placebo group, $24.7 \%$ of them had no cyst in ultrasonography. $13.5 \%$ of patients in green cardamom group improved and $10.8 \%$ of patients in placebo group improved in terms of cyst size and number decreasing in which these differences were significant between the two studied groups $(P=0.031)$ (Fig. 2). 
Table 2 Dietary intake, anthropometric and biochemical indices among women with PCOS before and after intervention with green cardamom

\begin{tabular}{|c|c|c|c|c|c|c|c|c|}
\hline \multirow[t]{2}{*}{ Variables } & \multicolumn{2}{|c|}{ Green cardamom $(n=99)$} & \multirow[t]{2}{*}{ P1 } & \multicolumn{2}{|l|}{ Placebo $(n=95)$} & \multirow[t]{2}{*}{ P1 } & \multirow[t]{2}{*}{$\mathrm{P} 2$} & \multirow[t]{2}{*}{ P3 } \\
\hline & Before & After & & Before & After & & & \\
\hline Age, year & $32.99 \pm 5.57 *$ & & & $33.81 \pm 5.42$ & & & 0.073 & \\
\hline Weight, $\mathrm{kg}$ & $86.26 \pm 10$ & $79.65 \pm 10.98$ & $<0.001$ & $87.38 \pm 10.75$ & $81.48 \pm 12.40$ & $<0.001$ & 0.506 & 0.156 \\
\hline $\mathrm{WC}, \mathrm{cm}$ & $113.01 \pm 12.55$ & $106.30 \pm 9.33$ & $<0.001$ & $113.57 \pm 7.89$ & $108.18 \pm 9.88$ & $<0.001$ & 0.683 & 0.366 \\
\hline BMI, $\mathrm{kg} / \mathrm{m}^{2}$ & $34.78 \pm 3.39$ & $32.13 \pm 4.46$ & $<0.001$ & $35.18 \pm 5.16$ & $32.86 \pm 5.95$ & $<0.001$ & 0.989 & 0.015 \\
\hline Body fat, $\%$ & $46.12 \pm 2.72$ & $44.48 \pm 2.53$ & $<0.001$ & $47.13 \pm 3.11$ & $44.76 \pm 3.06$ & $<0.001$ & 0.151 & 0.036 \\
\hline Energy (kcal/day) & $3424.24 \pm 769.51$ & $2216.61 \pm 590.47$ & $<0.001$ & $3101.48 \pm 748.6$ & $2189.48 \pm 633.14$ & $<0.001$ & 0.5 & 0.801 \\
\hline Protein (g/day) & $121.03 \pm 37.11$ & $84.81 \pm 24.01$ & $<0.001$ & $106.68 \pm 32.09$ & $84.22 \pm 25.21$ & $<0.001$ & 0.158 & 0.91 \\
\hline Carbohydrate (g/day) & $500.17 \pm 140.71$ & $348.57 \pm 102.75$ & $<0.001$ & $460.17 \pm 138.14$ & $342.79 \pm 113.71$ & $<0.001$ & 0.704 & 0.591 \\
\hline Fat (g/day) & $106.82 \pm 33.75$ & $56.13 \pm 18.54$ & $<0.001$ & $93.91 \pm 28.19$ & $55.89 \pm 20.92$ & $<0.001$ & 0.193 & 0.639 \\
\hline $\mathrm{TSH}, \mu \mathrm{IU} / \mathrm{mL}$ & $2.86 \pm 0.21$ & $2.88 \pm 0.27$ & 0.469 & $2.89 \pm 0.25$ & $2.89 \pm 0.34$ & 0.787 & 0.704 & 0.724 \\
\hline Androstenedione, $\mathrm{ng} / \mathrm{mL}$ & $2.09 \pm 1.81 *$ & $1.68 \pm 0.25$ & $<0.001$ & $1.97 \pm 0.32$ & $1.85 \pm 0.24$ & 0.013 & 0.121 & $<0.001$ \\
\hline DHEA, ng/dL & $321.27 \pm 62.38$ & $282.97 \pm 64.54$ & $<0.001$ & $363.39 \pm 76.01$ & $383.06 \pm 48.71$ & 0.109 & 0.198 & 0.033 \\
\hline Prolactin, $\mathrm{ng} / \mathrm{mL}$ & $8.44 \pm 4.22$ & $8.54 \pm 3.87$ & 0.884 & $7.78 \pm 4.09$ & $7.98 \pm 3.65$ & 0.747 & 0.647 & 0.594 \\
\hline Testosterone, $\mathrm{ng} / \mathrm{mL}$ & $1.24 \pm 0.21$ & $1.19 \pm 0.3$ & 0.188 & $1.35 \pm 0.21$ & $1.35 \pm 0.25$ & 0.968 & 0.99 & 0.016 \\
\hline LH, IU/L & $6.31 \pm 1.41$ & $3.36 \pm 1.41$ & $<0.001$ & $5.94 \pm 2.28$ & $5.95 \pm 1.84$ & 0.774 & $<0.001$ & $<0.001$ \\
\hline FSH, IU/L & $1.82 \pm 0.74$ & $2.77 \pm 1.42$ & $<0.001$ & $1.32 \pm 0.84$ & $1.43 \pm 0.95$ & 0.768 & 0.283 & $<0.001$ \\
\hline $\mathrm{SHBG}, \mathrm{nm} / \mathrm{L}$ & $27.77 \pm 10.63$ & $29.42 \pm 10.51$ & 0.261 & $33.12 \pm 10.17$ & $34.79 \pm 10.59$ & 0.251 & 0.726 & 0.829 \\
\hline
\end{tabular}

P1: $P$ values denote significance of within-group changes

P2: $P$ values denote significance of between-group difference in the baseline

P3: $P$ values denote significance of between-group difference after intervention

*All presented values are means $\pm \mathrm{SD}$

*Significant difference within group throughout the study $(P<0.05$, paired samples $t$-test or Wilcoxon)

*Significant difference between groups throughout the study $(P<0.05$, independent samples $t$-test or $U$ Mann-Whitney)

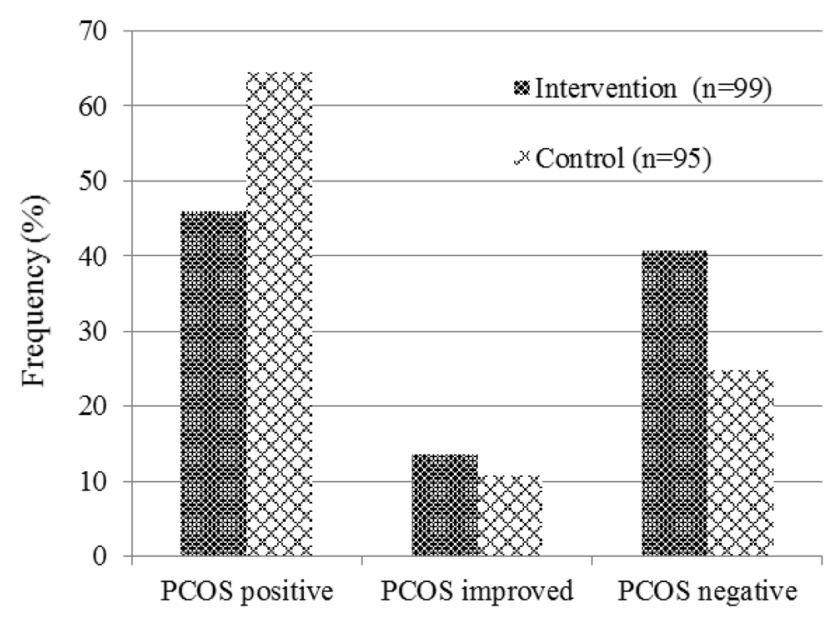

Fig. 2 Improvement of PCOS after intervention with green cardamom plus low-calorie diet

Our study indicated that the mean of weight, BMI, WC, and BFM was significantly decreased after intervention in both studied groups due to adherence to the low-calorie diet $(P<0.001)$. Among androgen hormones, androstenedione, DHEA, and LH were significantly decreased in the intervention group $(P<0.001)$. FSH was significantly decreased after supplementation with green cardamom plus low-calorie $\operatorname{diet}(P<0.001)$ (Table 2).

This study showed that green cardamom plus lowcalorie diet significantly reduces TNF- $\alpha$, IL-6, and CRP serum $(P<0.001)$ (Table 3 and Fig. $3 \mathrm{a}, \mathrm{c}, \mathrm{e})$. In addition, we observed that the expression level of TNF- $\alpha$ and $C R P$ genes was significantly decreased after the intervention with green cardamom plus low-calorie diet $(P<0.001)$ (Table 3 and Fig. 3b, f).

\section{Discussion}

This 16-week trial demonstrated that green cardamom supplementation can improve anthropometric indices, androgen hormones, and inflammatory factors levels and gene expression in PCOS patients with low-calorie diet. PCOS patients suffer from low-grade systemic inflammation that potentially affects their disease progression [22]. To the best of our knowledge, this is the first study that has evaluated the effects of green cardamom on anthropometric indices, androgen hormones, and inflammatory factors levels and gene expression in PCOS patients with low-calorie diet. 
Table 3 Effects of the green cardamom on inflammatory factors and genes

\begin{tabular}{|c|c|c|c|c|c|c|c|c|}
\hline \multirow[t]{2}{*}{ Genes } & \multicolumn{2}{|c|}{ Intervention $(n=99)$} & \multirow[t]{2}{*}{ P1 } & \multicolumn{2}{|l|}{ Control $(n=95)$} & \multirow[t]{2}{*}{$\mathrm{P} 1$} & \multirow[t]{2}{*}{$\mathrm{P} 2$} & \multirow[t]{2}{*}{ P3 } \\
\hline & Before & After & & Before & After & & & \\
\hline $\mathrm{TNF}-\alpha, p g / \mathrm{mL}$ & $7.34 \pm 0.84$ & $5.52 \pm 1.00$ & $<0.001$ & $6.62 \pm 0.51$ & $6.58 \pm 1.58$ & 0.456 & 0.001 & $<0.001$ \\
\hline IL-6, pg/mL & $2.65 \pm 1.61$ & $1.53 \pm 1.29$ & $<0.001$ & $2.28 \pm 1.41$ & $2.17 \pm 1.42$ & 0.76 & 0.007 & $<0.001$ \\
\hline hs-CRP, mg/mL & $1.56 \pm 0.2$ & $1.41 \pm 0.39$ & $<0.001$ & $1.50 \pm 0.18$ & $1.51 \pm 0.18$ & 1 & 0.673 & 0.139 \\
\hline$T N F-\alpha$ & $6.76 \pm 4.47$ & $4.16 \pm 5.75$ & $<0.001$ & $5.6 \pm 11$ & $5.42 \pm 5.06$ & 0.07 & $<0.001$ & 0.071 \\
\hline$I L-6 r$ & $10.39 \pm 11.51$ & $9.88 \pm 10.46$ & 0.657 & $10.54 \pm 12.39$ & $10.38 \pm 8.44$ & 0.886 & 0.857 & 0.17 \\
\hline$C R P$ & $10.6 \pm 13.3$ & $4.1 \pm 4.36$ & 0.044 & $8.91 \pm 13.09$ & $8.58 \pm 8.61$ & 0.203 & 0.335 & 0.001 \\
\hline
\end{tabular}

P1: $P$ values denote significance of within-group changes

P2: $P$ values denote significance of between-group difference in the baseline

P3: $P$ values denote significance of between-group difference after intervention

*All presented values are means $\pm \mathrm{SD}$

*Significant difference within group throughout the study $(P<0.05$, Wilcoxon)

*Significant difference between groups throughout the study $(P<0.05, U$ Mann-Whitney $)$

We found that weight, BMI, WC, and BFM were significantly reduced in both two studied groups. However, Yaghooblou et al. [23] reported that weight, BMI, WC, were significantly decreased compared to control group after administering $3 \mathrm{~g} /$ day of cardamom for 2 months. The high content of isoflavones and flavonoids in the green cardamom reduces the storage of adipose tissue [24]. Significant changes in the placebo group appear to be due to the lowcalorie diet in this group.

On the other hand, we observed improvement in the level of endocrine hormones including androstenedione, DHEA, and $\mathrm{LH}$, as well as FSH, after intervention with $3 \mathrm{~g} /$ day green cardamom. Obesity, especially abdominal obesity, as well as insulin resistance exacerbate hyperandrogenism. Obesity in PCOS patients causes the release of free fatty acids that increase insulin resistance. Increased body fat and insulin resistance act synergistically to upset the balance of endocrine hormones [5, 25]. Green cardamom has a role in reducing fat storage due to its anti-inflammatory effects, and its consumption seems to lead to an improvement in the hormonal status.

In the current study, we found that TNF- $\alpha$, IL-6, and CRP serum levels were significantly decreased. Besides, the expression of $T N F-\alpha$ and $C R P$ genes was significantly down-regulated after green cardamom supplementation in PCOS patients. Zahedi et al. [26] showed in their practice that administration of $3 \mathrm{~g} /$ day green cardamom for 10 weeks could reduce inflammatory factors in type two diabetes $(P<0.05)$. Souissi's study at the cellular level showed that both fruits and seeds of green cardamom exhibit antiinflammatory effects [14]. In contrast, Azimi et al. [24] did not observe any difference in CRP serum after 8 weeks of intervention with green cardamom on type two diabetic patients $(P=0.92)$. On the other hand, the randomized clinical trial results on pre-diabetic women with overweight and obesity showed that green cardamom supplementation leads to a significant decrease in CRP serum $(P=0.02)$, while no difference was observed between TNF- $\alpha$ and IL- 6 of them before and after the intervention $(P=0.1,0.4$, respectively) [17]. In addition, the study by Daneshi-Maskooni et al. [27] showed that administration of green cardamom decreased all three mentioned inflammatory factors in non-alcoholic fatty liver patients.

According to the present study's finding, the possible mechanisms of the expression of down-regulating inflammatory genes and consequent inflammatory cytokine reducing, and its effect on the improvement of PCOS patients are as follows: TNF- $\alpha$ is a pro-inflammatory biomarker which is abundantly produced in adipose tissue macrophages (Fig. 4). High levels of TNF- $\alpha$ increase dyslipidemia, insulin resistance, and secretion of IL-6 [27, 28] and could cause the proliferation of theca interna cells, which leads to hyperplasia and an increased number of follicles. TNF- $\alpha$ can also decrease ovulation by reducing progesterone production. It prevents the ovum's release by autophagy and apoptosis of granulosa cells and estrogen reduction [1, 7]. IL-6 is an endocrine, inflammatory cytokine produced in acute and chronic inflammation in many body cells such as adipose tissue [27]. High IL-6 level is associated with obesity, insulin resistance, hyperandrogenism, and ovarian dysfunction $[1,7,28]$. The evidence shows that CRP is associated with increased adipose tissue, obesity, dyslipidemia, and insulin resistance and is high in PCOS patients [1, 12]. Green cardamom is an excellent source of essential oils, flavonoids, 
TNF-a, pg/ml

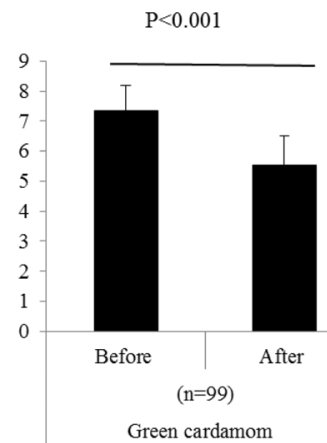

a

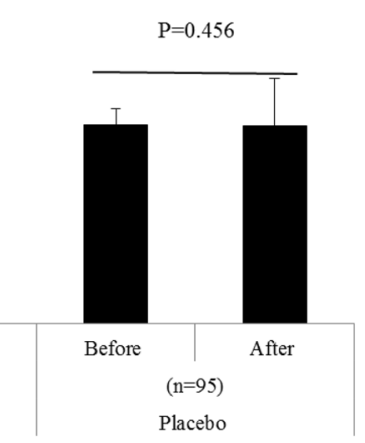

IL-6, pg/ml $\mathrm{P}<0.001$

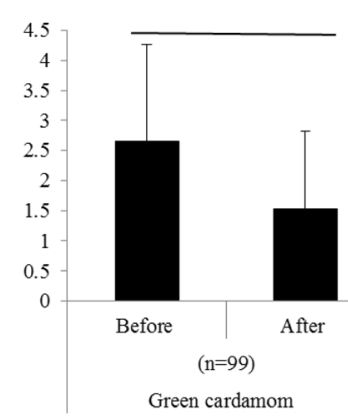

C

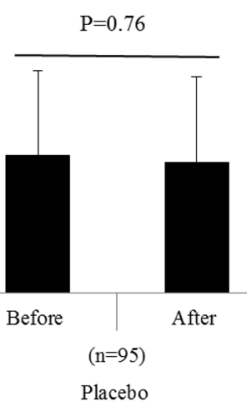

TNF-a

b

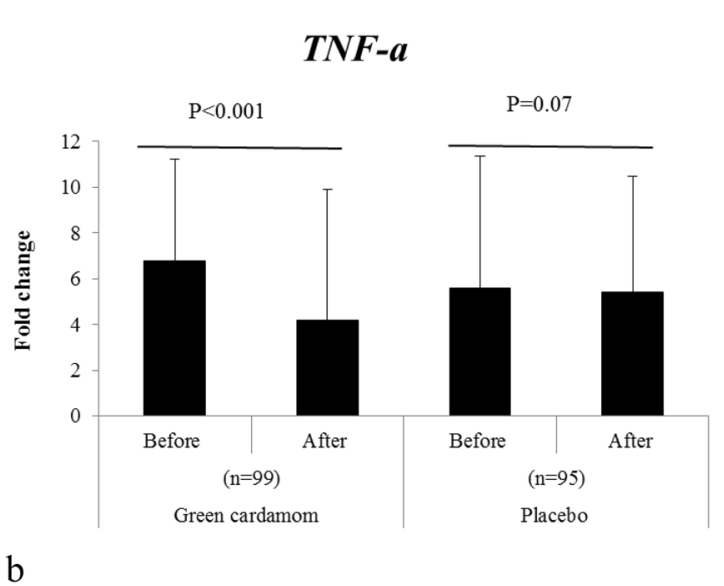

$I L-6 r$

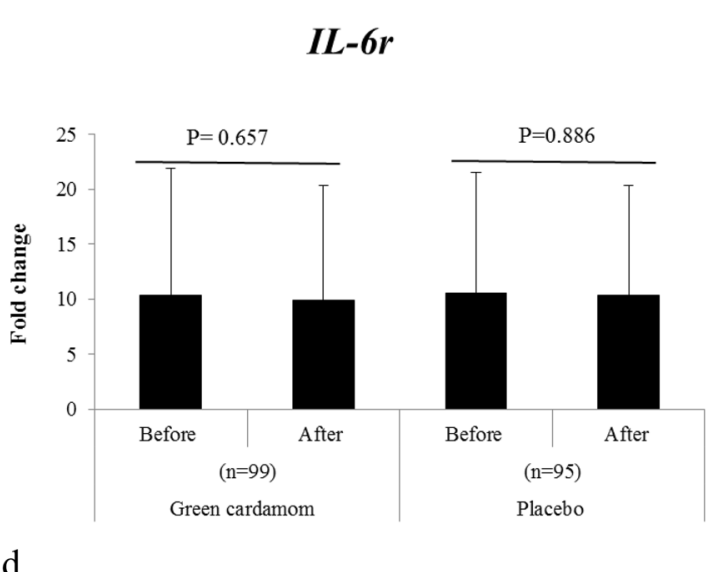

d

\section{hs-CRP, mg/ml}

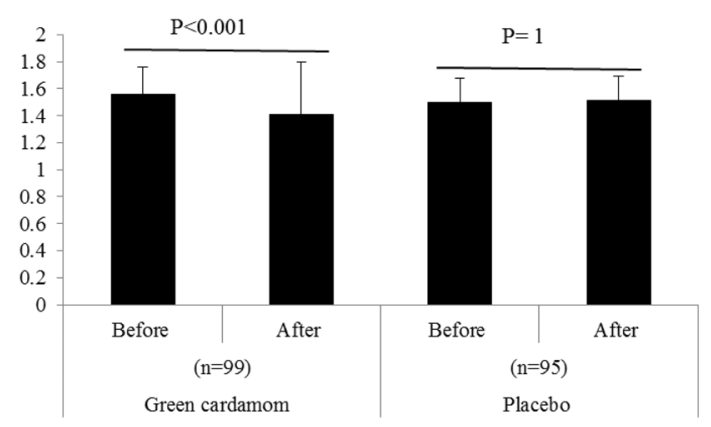

e

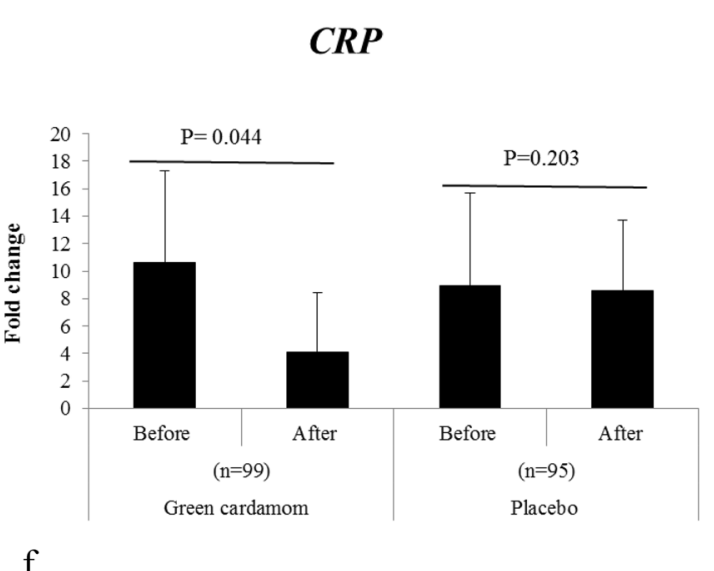

Fig. 3 Changes of inflammatory markers and genes including: a TNF- $\alpha$ serum, b TNF- $\alpha$ gene, $\mathbf{c}$ IL-6 serum, d $I L-6 r$ gene, e CRP serum, and f $C R P$ gene before and after intervention with green cardamom

polyphenols, and sterols with analgesic, anti-inflammatory, antimicrobial and antioxidant properties [29]. The findings of our study showed the effect of the green cardamom on reducing inflammation by down-regulated the expression of inflammatory genes. Furthermore, weight loss by reducing adipose tissue with green cardamom consumption synergistically reduces these inflammatory factors and improves the disease. 


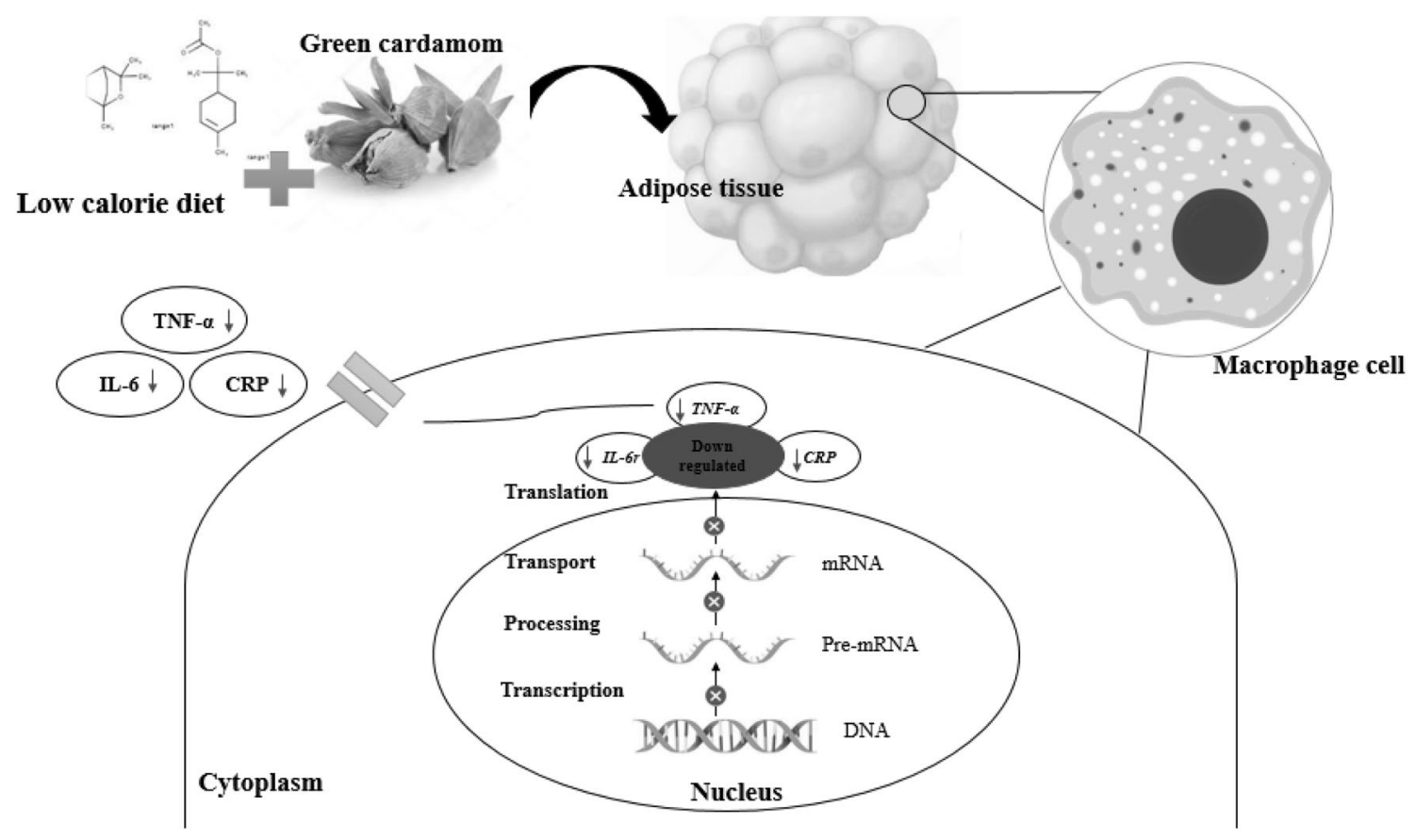

Fig. 4 Effects of the low-calorie diet plus green cardamom on inflammatory factors and genes

\section{Strength and limits}

This trial was the first study that investigated the effect of green cardamom on reducing inflammatory factor levels and their gene expression. Although the statistical analysis controlled for dietary intake and other potential confounders, low-caloric diet might have been related to the reduction in pro-inflammatory cytokines by decreasing body fat.

This study has several limitations. First, sports clubs were closed because of the coronavirus at the time of the sampling. Therefore, the subjects could not perform appropriate exercises to contribute to the weight loss diet. Second, some samples were excluded from the study due to coronavirus infection, although we had considered a larger number of samples. Third, due to financial constraints, we could not measure more inflammatory factors and genes. Fourth, diet and physical activity were self-reported. Further studies are needed to increase our knowledge about and ensure the safety and efficacy of cardamom.

\section{Conclusion}

Overall, the findings of our study showed that the administration of $3 \mathrm{~g} /$ day green cardamom was associated with an improvement in anthropometric indices, endocrine hormones, and inflammatory cytokines in obese PCOS women with lowcalorie diet. Furthermore, the expression of inflammatory genes was significantly down-regulated after consuming the green cardamom supplement.

\section{What is already known on this subject?}

Polycystic ovary syndrome (PCOS) is a common endocrine disease among women that causes infertility. This syndrome is commonly associated with obesity, hirsutism, menstrual irregularities, and insulin resistance. Evidence also suggests that the disease is associated with a pro-inflammatory condition. Therefore, nutritional interventions with weight loss and inflammatory factors seem to be a suitable strategy in reducing its progression.

\section{What does this study add?}

The current well-designed trial states that the consumption of green cardamom and adherence to low-calorie diet in obese PCOS women could improve anthropometric indices, endocrine hormones, and inflammatory cytokines. Additionally, the expression of inflammatory genes was significantly down-regulated after consuming the green cardamom supplement. 
Acknowledgements We wish to thank and acknowledge the valuable contribution of all women in this study and especially want to acknowledge Kermanshah University of Medical Science for financial support.

Author contributions $\mathrm{SM}$ and $\mathrm{SCh}$ contributed in conception and design of the research; NHE, NH, MG, EM, and FK contributed to data collection; SM and SCh contributed to the acquisition, analysis, and interpretation of the data; SM, SCh, and YP contributed to draft the manuscript. SM, Sch, and NR contributed to revise the manuscript. All authors are in agreement with the manuscript and declare that the content has not been published elsewhere.

Funding This study was supported by Kermanshah University of Medical Science (Grant No: 990412).

Availability of data and materials Data will be available upon request from the corresponding author.

Conflict of interest On behalf of all co-authors, the corresponding author states that there is no conflict of interest.

Ethical approval All procedures performed in studies involving human participants were in accordance with the ethical standards of the institutional and/or national research committee and with the 1964 Helsinki declaration and its later amendments or comparable ethical standards. This study was approved by the Ethics Committee of Kermanshah University of Medical Sciences (ethics approval number: IR.KUMS. REC.1399.375) and registered with the Iranian Clinical Trials Registry (registration number: IRCT20200608047697N1). 1 August, 2020; https://www.irct.ir/trial/48748.

Informed consent Written informed consent was obtained from each studied subject after explaining the purpose of the study. The right of subjects to withdraw from the study at any time and subjects information is reserved and will not be published.

\section{References}

1. Rostamtabar M, Esmaeilzadeh S, Tourani M, Rahmani A, Baee M, Shirafkan F, Saleki K, Mirzababayi SS, Ebrahimpour S, Nouri HR (2021) Pathophysiological roles of chronic low-grade inflammation mediators in polycystic ovary syndrome. J Cell Physiol 236(2):824-838. https://doi.org/10.1002/jcp.29912

2. Popovic M, Sartorius G, Christ-Crain M (2019) Chronic lowgrade inflammation in polycystic ovary syndrome: is there a (patho)-physiological role for interleukin-1? Seminars in immunopathology, vol 4. Springer, pp 447-459

3. Shorakae S, Ranasinha S, Abell S, Lambert G, Lambert E, de Courten B, Teede H (2018) Inter-related effects of insulin resistance, hyperandrogenism, sympathetic dysfunction and chronic inflammation in PCOS. Clin Endocrinol 89(5):628-633. https:// doi.org/10.1111/cen.13808

4. Patel S (2018) Polycystic ovary syndrome (PCOS), an inflammatory, systemic, lifestyle endocrinopathy. J Steroid Biochem Mol Biol 182:27-36. https://doi.org/10.1016/j.jsbmb.2018.04.008

5. Zeng X, Xie Y-j, Liu Y-t, Long S-1, Mo Z-c (2020) Polycystic ovarian syndrome: correlation between hyperandrogenism, insulin resistance and obesity. Clin Chim Acta 502:214-221. https://doi. org/10.1016/j.cca.2019.11.003

6. Chow LS, Mashek DG, Wang Q, Shepherd SO, Goodpaster BH, Dubé JJ (2017) Effect of acute physiological free fatty acid elevation in the context of hyperinsulinemia on fiber type-specific
IMCL accumulation. J Appl Physiol 123(1):71-78. https://doi.org/ 10.1152/japplphysiol.00209.2017

7. Mohammadi S, Kayedpoor P, Karimzadeh-Bardei L, Nabiuni M (2017) The effect of curcumin on TNF- $\alpha$, IL- 6 and CRP expression in a model of polycystic ovary syndrome as an inflammation state. J Reprod Infertil 18(4):352

8. Guo R, Zheng Y, Yang J, Zheng N (2015) Association of TNFalpha, IL-6 and IL-1beta gene polymorphisms with polycystic ovary syndrome: a meta-analysis. BMC Genet 16(1):1-13. https:// doi.org/10.1186/s12863-015-0165-4

9. Rafiq S, Frayling T, Murray A, Hurst A, Stevens K, Weedon M, Henley W, Ferrucci L, Bandinelli S, Corsi A (2007) A common variant of the interleukin 6 receptor (IL-6r) gene increases IL-6r and IL-6 levels, without other inflammatory effects. Genes Immun 8(7):552-559. https://doi.org/10.1038/sj.gene.6364414

10. Chen L, Zhang Z, Huang J, Jin M (2018) Association between rs 1800795 polymorphism in the interleukin- 6 gene and the risk of polycystic ovary syndrome: a meta-analysis. Medicine. https:// doi.org/10.1097/MD.0000000000011558

11. Sheu WH-H, Wang W-C, Wu K-D, He C-T, Hwu C-M, Quertermous T, Hsieh W-S, Lee W-J, Ting C-T, Chen Y-DI (2017) CRPlevel-associated polymorphism rs 1205 within the CRP gene is associated with 2-hour glucose level: the SAPPHIRe study. Sci Rep 7(1):1-8. https://doi.org/10.1038/s41598-017-08696-2

12. Blumenfeld $Z$ (2019) The possible practical implication of high CRP levels in PCOS. SAGE Publications Sage UK, London

13. Ashokkumar K, Murugan M, Dhanya M, Warkentin TD (2020) Botany, traditional uses, phytochemistry and biological activities of cardamom [Elettaria cardamomum (L.) Maton] - a critical review. J Ethnopharmacol 246:112244. https://doi.org/10.1016/j. jep.2019.112244

14. Souissi M, Azelmat J, Chaieb K, Grenier D (2020) Antibacterial and anti-inflammatory activities of cardamom (Elettaria cardamomum) extracts: potential therapeutic benefits for periodontal infections. Anaerobe 61:102089. https://doi.org/10.1016/j.anaer obe.2019.102089

15. Daneshi-Maskooni M, Keshavarz SA, Mansouri S, Qorbani M, Alavian SM, Badri-Fariman M, Jazayeri-Tehrani SA, Sotoudeh G (2017) The effects of green cardamom on blood glucose indices, lipids, inflammatory factors, paraxonase-1, sirtuin-1, and irisin in patients with nonalcoholic fatty liver disease and obesity: study protocol for a randomized controlled trial. Trials 18(1):1-9. https://doi.org/10.1186/s13063-017-1979-3

16. Boutron I, Moher D, Altman DG, Schulz KF, Ravaud P (2008) Extending the CONSORT statement to randomized trials of nonpharmacologic treatment: explanation and elaboration. Ann Inter Med 148(4):295-309. https://doi.org/10.7326/0003-4819-148-4200802190-00008-w1

17. Kazemi S, Yaghooblou F, Siassi F, Rahimi Foroushani A, Ghavipour M, Koohdani F, Sotoudeh G (2017) Cardamom supplementation improves inflammatory and oxidative stress biomarkers in hyperlipidemic, overweight, and obese pre-diabetic women: a randomized double-blind clinical trial. J Sci Food Agric 97(15):5296-5301. https://doi.org/10.1002/jsfa.8414

18. Aghasi M, Ghazi-Zahedi S, Koohdani F, Siassi F, Nasli-Esfahani E, Keshavarz A, Qorbani M, Khoshamal H, Salari-Moghaddam A, Sotoudeh G (2018) The effects of green cardamom supplementation on blood glucose, lipids profile, oxidative stress, sirtuin- 1 and irisin in type 2 diabetic patients: a study protocol for a randomized placebo-controlled clinical trial. BMC Complement Altern Med 18(1):18. https://doi.org/10.1186/s12906-017-2068-6

19. Daneshi-Maskooni M, Keshavarz SA, Qorbani M, Mansouri S, Alavian SM, Badri-Fariman M, Jazayeri-Tehrani SA, Sotoudeh G (2019) Green cardamom supplementation improves serum irisin, glucose indices, and lipid profiles in overweight or obese nonalcoholic fatty liver disease patients: a double-blind randomized 
placebo-controlled clinical trial. BMC Complement Altern Med 19(1):59. https://doi.org/10.1186/s12906-019-2465-0

20. Ghaffarpour M, Houshiar-Rad A, Kianfar H (1999) The manual for household measures, cooking yields factors and edible portion of foods, vol 7. Nashre Olume Keshavarzy, Tehran, p 213

21. Mahan L, Raymond J (2016) Krause's food \& the nutrition care process; clinical: biochemical, physical, and functional assessment, 14th edn. Elsevier Health Sciences

22. Tosatti JA, Alves MT, Cândido AL, Reis FM, Araújo VE, Gomes KB (2020) Influence of n-3 fatty acid supplementation on inflammatory and oxidative stress markers in patients with polycystic ovary syndrome: a systematic review and meta-analysis. Br J Nutr. https://doi.org/10.1017/S0007114520003207

23. Fatemeh Y, Siassi F, Rahimi A, Koohdani F, Doostan F, Qorbani M, Sotoudeh G (2017) The effect of cardamom supplementation on serum lipids, glycemic indices and blood pressure in overweight and obese pre-diabetic women: a randomized controlled trial. Diabetes Metab Syndr 16(1):40

24. Azimi P, Ghiasvand R, Feizi A, Hariri M, Abbasi B (2014) Effects of cinnamon, cardamom, saffron, and ginger consumption on markers of glycemic control, lipid profile, oxidative stress, and inflammation in type 2 diabetes patients. RDS 11(3):258. https:// doi.org/10.1900/RDS.2014.11.258

25. Sirotkin AV, Fabian D, Babel’ová J, Vlčková R, Alwasel S, Harrath AH (2018) Body fat affects mouse reproduction, ovarian hormone release, and response to follicular stimulating hormone. Reprod Biol 18(1):5-11. https://doi.org/10.1186/s 12944-017-0539-x

26. Zahedi SG, Koohdani F, Qorbani M, Siassi F, Keshavarz A, NasliEsfahani E, Aghasi M, Khoshamal H, Sotoudeh G (2020) The effects of green cardamom supplementation on blood pressure and endothelium function in type 2 diabetic patients: a study protocol for a randomized controlled clinical trial. Medicine 99(18):e11005. https://doi.org/10.1097/MD.0000000000011005

27. Daneshi-Maskooni M, Keshavarz SA, Qorbani M, Mansouri S, Alavian SM, Badri-Fariman M, Jazayeri-Tehrani SA, Sotoudeh G (2018) Green cardamom increases Sirtuin-1 and reduces inflammation in overweight or obese patients with non-alcoholic fatty liver disease: a double-blind randomized placebo-controlled clinical trial. Nutr Metab 15(1):1-12. https://doi.org/10.1186/ s12986-018-0297-4

28. Cardoso NS, Ribeiro VB, Dutra SGV, Ferriani RA, Gastaldi AC, Araújo JEd, Souza HCDD (2020) Polycystic ovary syndrome associated with increased adiposity interferes with serum levels of TNF-alpha and IL-6 differently from leptin and adiponectin. Arch Endocrinol Metab 64(1):4-10. https://doi.org/10.20945/ 2359-3997000000197

29. Rahman MM, Alam MN, Ulla A, Sumi FA, Subhan N, Khan T, Sikder B, Hossain H, Reza HM, Alam MA (2017) Cardamom powder supplementation prevents obesity, improves glucose intolerance, inflammation and oxidative stress in liver of high carbohydrate high fat diet induced obese rats. Lipids Health Dis 16(1):151. https://doi.org/10.1186/s12944-017-0539-x

Publisher's Note Springer Nature remains neutral with regard to jurisdictional claims in published maps and institutional affiliations. 